\title{
Nectar sugars and bird visitation define a floral niche for basidiomycetous yeast on the Canary Islands
}

\author{
Moritz Mittelbach ${ }^{1 *}$, Andrey M Yurkov ${ }^{2}$, Daniele Nocentini ${ }^{3}$, Massimo Nepi ${ }^{3}$, Maximilian Weigend ${ }^{4}$ \\ and Dominik Begerow ${ }^{1}$
}

\begin{abstract}
Background: Studies on the diversity of yeasts in floral nectar were first carried out in the late $19^{\text {th }}$ century. A narrow group of fermenting, osmophilous ascomycetes were regarded as exclusive specialists able to populate this unique and species poor environment. More recently, it became apparent that microorganisms might play an important role in the process of plant pollination. Despite the importance of these nectar dwelling yeasts, knowledge of the factors that drive their diversity and species composition is scarce.

Results: In this study, we linked the frequencies of yeast species in floral nectars from various host plants on the Canary Islands to nectar traits and flower visitors. We estimated the structuring impact of pollination syndromes (nectar volume, sugar concentration and sugar composition) on yeast diversity.

The observed total yeast diversity was consistent with former studies, however, the present survey yielded additional basidiomycetous yeasts in unexpectedly high numbers. Our results show these basidiomycetes are significantly associated with ornithophilous flowers. Specialized ascomycetes inhabit sucrose-dominant nectars, but are surprisingly rare in nectar dominated by monosaccharides.

Conclusions: There are two conclusions from this study: (i) a shift of floral visitors towards ornithophily alters the likelihood of yeast inoculation in flowers, and (ii) low concentrated hexose-dominant nectar promotes colonization of flowers by basidiomycetes. In the studied floral system, basidiomycete yeasts are acknowledged as regular members of nectar. This challenges the current understanding that nectar is an ecological niche solely occupied by ascomycetous yeasts.
\end{abstract}

Keywords: Basidiomycetes, Bird pollination, Boraginaceae, Canary Islands, Nectar-dwelling yeast

\section{Background}

Several recent studies have invoked a resurgent interest in the importance of pollination to plant reproductive success and fertility [1,2]. Herrera et al. [3] and Vannette et al. [4] presented the first evidence for microbially-mediated impacts on plant pollination and fecundity by nectar dwelling yeast and bacteria, respectively. Nectar-dwelling unicellular fungi (yeasts) have fascinated researchers for over a hundred years. For example, ascomycetous yeasts, namely Metschnikowia reukaufii Pitt \& M.W. Mill. and

\footnotetext{
* Correspondence: moritz.mittelbach@rub.de

'Department of Geobotany, - LS Evolution \& Biodiversity of Plants, Ruhr-University Bochum, ND 1/150 / Universitaetsstr. 150, 44780 Bochum, Germany

Full list of author information is available at the end of the article
}

Metschnikowia gruessii Gim.-Jurado, were known since the late nineteenth century as common inhabitants of floral nectar in various host flowers [5]. Subsequent studies addressed the distribution [6], ecology [7-9], and physiological properties [10-12] of these species. Research in the past years added knowledge on functionality [13], population structure [14] and epigenetic variability of Metschnikowia reukaufii [15]. Studies on flowers all over the world strengthened the impression of a narrow and highly specific nectarivorous yeast community [16-18], which may consist up to $85 \%$ of fast growing ascomycetous specialists [19], adapted to sugar rich, temporally and spatially fragmented nectar environments.

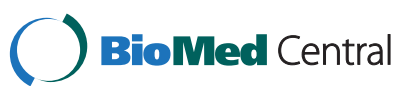

(c) 2015 Mittelbach et al.; licensee BioMed Central. This is an Open Access article distributed under the terms of the Creative Commons Attribution License (http://creativecommons.org/licenses/by/4.0), which permits unrestricted use, distribution, and reproduction in any medium, provided the original work is properly credited. The Creative Commons Public Domain Dedication waiver (http://creativecommons.org/publicdomain/zero/1.0/) applies to the data made available in this article, unless otherwise stated. 
A broader diversity of yeast species has been regularly reported from nectar $[6,20,21]$, including both unicellular non-fermenting yeasts and yeast-like fungi [22,23]. Basidiomycetous yeasts are supposedly unable to persist in specific nectar environments based on their in vitro properties, such as growth preferences determined in culture media [24,25]. Thus, they are mostly regarded as autochthonous to non-flower habitats, such as plant surfaces or soils $[23,26,27]$ and their presence in floral nectar is usually believed to be a contamination from other neighboring substrates [24].

The nectar of individual flowers represent species-poor habitats $[18,19]$ characterized by both harsh physiological conditions [28] and strong species competition [29], which favors fast-growing microorganisms. The co-evolution of pollination syndromes between plants and flower visitors shaped a tremendous variety of floral habitats, which differ amongst other traits in nectar volume, and sugar concentration and composition [30,31]. Reukauf [7] and later Sandhu and Waraich [22] interpreted interspecific variation of nectar-traits as influential to nectar inhabiting fungi, however, Brysch-Herzberg [24] found no evidence for this in his exhaustive study. Since then, a few studies showed a correlation of main pollinator groups to yeast quantities $[17,32]$, but not to the incidence of yeast nor their diversity [33].

The floral traits adapted to different pollination strategies should impose both direct and indirect effects on nectar dwellers. For example, Herrera et al. [28] showed the highly concentrated, sucrose-dominant nectar of Helleborus foetidus L. may act as a selective environmental filter for arriving yeast inoculum, which explains the dominance of osmophilic ascomycetes in nectar [16,34]. Responses of yeast community structure to nectar properties have received little attention so far, although different taxonomic groups of yeasts often possess distinct physiological characteristics [35] and assimilation tests of yeast strains demonstrated wide inter-specific differences in the ability to utilize various mono-, di, trisaccharides, as well as polyoles, alcohols etc. as a sole carbon source [36,37]. As a consequence, changes in the yeast environment, such as nectar chemistry, are likely to favor the growth of different yeast species, thereby directly affecting yeast community composition through the alteration of osmotic pressure, $\mathrm{pH}$ or availability of a particular nutrient.

The adaptation to environmental (nectar) habitats is only one factor responsible for a successful establishment of yeast populations. Equally important should be the propagation, the dispersal, and the inoculation of yeasts into nectar habitats, which are in turn indirectly driven by floral traits. Since nectar-dwelling yeasts are predominantly vectored by flower visitors [38], the composition and visitation frequencies of pollinator communities might conceivably govern the composition and abundances of the yeastinoculum, respectively. Visitor anatomy and behavior should additionally impact yeast transfer and inoculation. Thus, a lower degree of visitor specialization signifies foraging on a wider variety of flowers or even food sources other than nectar. This might lead to a more heterogeneous pool of microorganisms, including ones normally not found in nectar, via the constant transfer of microorganisms between substrates [39].

To study the influence of pollination syndromes on nectar-dwelling yeasts, we analyzed floral nectars on the island of Tenerife, Spain. The Canary Islands provide a unique bird-pollination element [40], comprising opportunistic nectar feeding passerine birds [41]. Different evolutionary scenarios for the origin of ornithophily in Macaronesia have been proposed. Most likely, birdrelated floral traits are relictual in some plant groups and de-novo in others (see [42] for Discussion). Flowers adapted to bird pollination are generally characterized by large red to orange corollas, diurnal anthesis, the absence of scent and the provision of suitable landing platforms [43]. Nectars are expected to be abundant, highly dilute with a dominance of monosaccharides [44]. However, on the Canary Islands, morphological adaptations of ornithophilous flowers are inconsistent with entomophilous relatives, albeit, Canarian bird species tend to prefer hexose (monosaccharide-dominated) to sucrose (disaccharide-dominated) nectars [45]. The de novo adaptation of ornithophily to passerine birds after island colonization is expected for the plant genus Echium L. (Boraginaceae) [46], which developed rather generalistic pollination syndromes and a variety of mixed bird/insect pollination systems [47].

In the present study we aim to link nectarivorous yeast diversity to different pollination-syndromes, addressing the impacts of nectar traits (volume, sugar concentration and composition) and floral visitors (frequency and composition). We hypothesize that yeast communities should be determined by two different sets of parameters: (i) alterations in the floral habitat itself, such as nectar concentration, abundance, and sugar composition, and (ii) the yeast transfer conditions as a result of different flower visitor assemblages.

\section{Results}

\section{Yeast diversity}

From 480 sampled flowers, 183 (38\%) yielded culturable yeasts, resulting in 220 yeast isolates classified in 34 species (Additional file 1). A total of 13 (6\%) identified yeasts were singletons (species found in a single sample only). Colonization frequencies differed considerably between years (16\% in 2012 and 49\% in 2013) and host plants: only $20 \%$ of Isoplexis canariensis (2013) flowers contained culturable yeast, while flowers of Teucrium 
heterophyllum (2013) yielded the highest percentage (75\%). Metschnikowia reukaufii was the most frequent yeast species $(n=43)$, although only isolated in 2013 . The survey yielded 32 strains of Metschnikowia gruessii (7 in 2012 and 25 in 2013) and 20 strains of Cryptococcus carnescens (8 in 2012 and 12 in 2013). The Shannon index of yeast diversity ranged from 1.0 in Echium leucophaeum (2013) to 2.4 in Canarina canariensis (2013) (Table 1). Most widespread yeast taxa were Metschnikowia gruessii and Cryptococcus carnescens, each isolated from flowers of six different host plants during the two years. M. reukaufii was found in five, while Starmerella bombicola and Cryptococcus heimaeyensis were found in nectars of four host plants. Mean yeast species richness in single flowers was $1.2( \pm 0.4 \mathrm{SD})$ with 3 species as the maximum richness per flower. Total yeast counts (CFUs per flower) ranged from 1 to approx. 1000 colonies per flower, but varied considerably within and between yeast species, flowers, and host plants (Additional file 1).

\section{Nectar analysis}

Analysis of main nectar-sugars (sucrose, glucose, and fructose) revealed two major groups of host plants with either sucrose-dominant or hexose-dominant nectars (Table 1). Nectar-volumes ranged from $0.9 \mu \mathrm{l}( \pm 0.2 \mathrm{SD})$ in E. strictum to $22.7 \mu \mathrm{l}( \pm 17)$ in $C$. canariensis with sugar concentrations from $14.5 \%( \pm 2.6)$ in E. leucophaeum to $42.7 \%( \pm 10.4)$ in T. heterophyllum.

\section{Observations of floral visitors}

We observed a total of 7503 flower visits on the 4 focal Echium host species. Individual visitation rates differed between the observed species up to one magnitude (Table 1). Echium strictum received the highest visitation frequency in 2012 (0.00378 visits per flower per minute (v/f/min) $\pm 0.052 \mathrm{SD})$ and Echium simplex the lowest in $2013(0.00037 \pm 0.004)$. Most abundant pollinator groups were bumblebees $(0.0056 \pm 0.001)$, consisting almost exclusively of visits by Bombus canariensis Pérez. Visitors of the functional group of bees $(0.00485 \pm 0.001)$ were classified as members of the genera Megachile and Osmia, while honeybees were only present in Echium strictum in 2012 and account for only $21 \%$ of all bee visits. Flower visiting birds were identified either as Common Chiffchaff (Phylloscopus collybita Vieillot), Atlantic Canary (Serinus canaria L.), or Blue Tit (Parus caeruleus L.; only on Echium leucophaeum) and have been observed on Echium leucophaeum in $2012(0.0001 \pm 0.003)$ and on Echium simplex in $2013(0.00011 \pm 0.003)$.

\section{Yeast communities and pollination syndromes}

Nectar traits and visitation frequencies, in our study defined as pollination syndromes are correlated (Mantel test: $r=0.426 ; p<0.01)$. This correlation impedes separate analyses of their impacts on nectar dwelling yeast communities, although the ordination plot (Figure 1) suggests that yeast species frequencies are clearly structured by sampled nectar traits (axis 1) and flower visitors (axis 2). Consequently, frequencies of yeast isolation in our study are significantly driven by nectar sugar type (PERMANOVA: $R^{2}=0.179$, $p<0.05)$ although only Cys. capitatum seems to be significantly related to hexose dominant nectars (Figure 2). The nectar type also significantly discriminates the relative incidences of ascomycetous versus basidiomycetous yeasts in our study (Figure 3).

In addition to the nectar sugar concentration, frequencies of visitors to flowers (birds, bees, bumblebees) influence yeast diversity (Figure 4). Although specialized nectar dwelling members of the Metschnikowiaceae lineage are ubiquitous, they form a phylogenetically clustered node assemblage in flowers with high sugar concentrations and are responsible for a reduced functional yeast diversity (FDis) in this substrate. The occurrence of these yeasts varied substantially between sampling years, with most pronounced fluctuation being observed for $M$. reukaufii. Nonetheless, this species was isolated from a total of 27 samples of host plants, which were not part of the analysis in 2012.

\section{Discussion}

The overall diversity of yeast species determined in this study is consistent with results of previous studies [19,48]. However, our study yielded numerous basidiomycetous yeasts regularly isolated from flowers and identified as Cryptococcus carnescens, Cr. heimaeyensis, and Cystofilobasidium capitatum (Additional file 1). Our results show that yeast communities are significantly mediated by the type of flower visitor and by nectar sugar concentration (Figure 4). This confirmed former hypotheses of flower-trait mediated yeast communities $[7,22]$ and expanded the known effects of pollinator composition [17] and pollination syndromes [32] on the diversity and composition of yeast communities. Although floral traits and pollinator composition are naturally correlated, they may steer two different mechanisms of the yeast-colonization process, namely (i) the ability to grow in nectar and (ii) the probability of flower inoculation. Below, we discuss the two mechanisms in more details.

(i) Among other natural yeast harboring substrates, such as plant surfaces, fruits, and soils $[26,27,49]$, nectar habitats stand out by high sugar concentrations, microaerophilic conditions, low nitrogen levels, and the widespread presence of anti-microbial compounds [50]. As has been shown by Peay et al. [29], these environmental conditions may regulate the growth of nectar dwelling microorganisms, giving nectar a filtering property for inoculated colonizers. Our results supported this hypothesis, as increased sugar concentrations favored the 
Table 1 Sampled host plants, nectar traits, flower visitors, \& diversity index

\begin{tabular}{|c|c|c|c|c|c|c|c|c|c|c|c|c|}
\hline \multirow[b]{2}{*}{ Species } & \multirow[b]{2}{*}{ Year } & \multicolumn{5}{|c|}{ Nectar traits } & \multicolumn{5}{|c|}{ Visitation frequencies } & \multirow{2}{*}{$\begin{array}{l}\text { Yeast diversity } \\
\text { Shannon }\end{array}$} \\
\hline & & Sucrose & Fructose & Glucose & Volume & Concentration & Bumblebees & Bees & Flies & Birds & InvSimpson & \\
\hline Canarina & & $\%$ & & & $\mu L$ & $\%$ & \multicolumn{4}{|c|}{ visits/flower/minute } & $1 / \lambda$ & H \\
\hline C. canariensis & 2013 & 0.1 & 49.7 & 50.3 & 22.7 & 27.1 & 0.000000 & 0.000000 & 0.000000 & 0.003000 & 1.0 & 2.4 \\
\hline \multicolumn{13}{|l|}{ Echium } \\
\hline E. leucophaeum & 2012 & 8.7 & 45.5 & 42.7 & 4.5 & 14.5 & 0.000013 & 0.000258 & 0.000070 & 0.000077 & 2.3 & 1.7 \\
\hline E. leucophaeum & 2013 & & & & 6.3 & 17.2 & 0.000059 & 0.000257 & 0.000236 & 0.000000 & 2.4 & 1.0 \\
\hline E. plantagineum & 2012 & 70.3 & 13.6 & 16.1 & 1.5 & 18.6 & 0.001384 & 0.000316 & 0.000109 & 0.000000 & 1.6 & 1.7 \\
\hline E. plantagineum 1 & 2013 & & & & 2 & 19.2 & 0.000368 & 0.000247 & 0.000040 & 0.000000 & 2.2 & 1.3 \\
\hline E. plantagineum 2 & 2013 & & & & 1.9 & 19.5 & 0.000346 & 0.000284 & 0.000072 & 0.000000 & 2.4 & 1.9 \\
\hline E. simplex & 2013 & 4.4 & 45.7 & 49 & 8.8 & 14.6 & 0.000023 & 0.000222 & 0.000007 & 0.000107 & 2.1 & 1.9 \\
\hline E. strictum & 2012 & 67.2 & 13.9 & 18.3 & 1.2 & 24.9 & 0.000900 & 0.002742 & 0.000135 & 0.000000 & 1.7 & 1.7 \\
\hline E. strictum & 2013 & & & & 0.9 & 24.8 & 0.002863 & 0.000800 & 0.000139 & 0.000000 & 1.6 & 1.9 \\
\hline \multicolumn{13}{|l|}{ Isoplexis } \\
\hline 1. canariensis & 2013 & 0.1 & 42.9 & 55.7 & 5.4 & 31.1 & 0.000000 & 0.000000 & 0.000000 & 0.011000 & 1.0 & 1.6 \\
\hline \multicolumn{13}{|l|}{ Lavatera } \\
\hline L. acerifolia & 2013 & 0.1 & 48.7 & 51.3 & 3.5 & 42.7 & NA & NA & NA & NA & NA & 1.2 \\
\hline \multicolumn{13}{|l|}{ Teucrium } \\
\hline T. heterophyllum & 2013 & 78.9 & 10.7 & 10.4 & 3.5 & 42.7 & NA & NA & NA & NA & NA & 1.1 \\
\hline
\end{tabular}




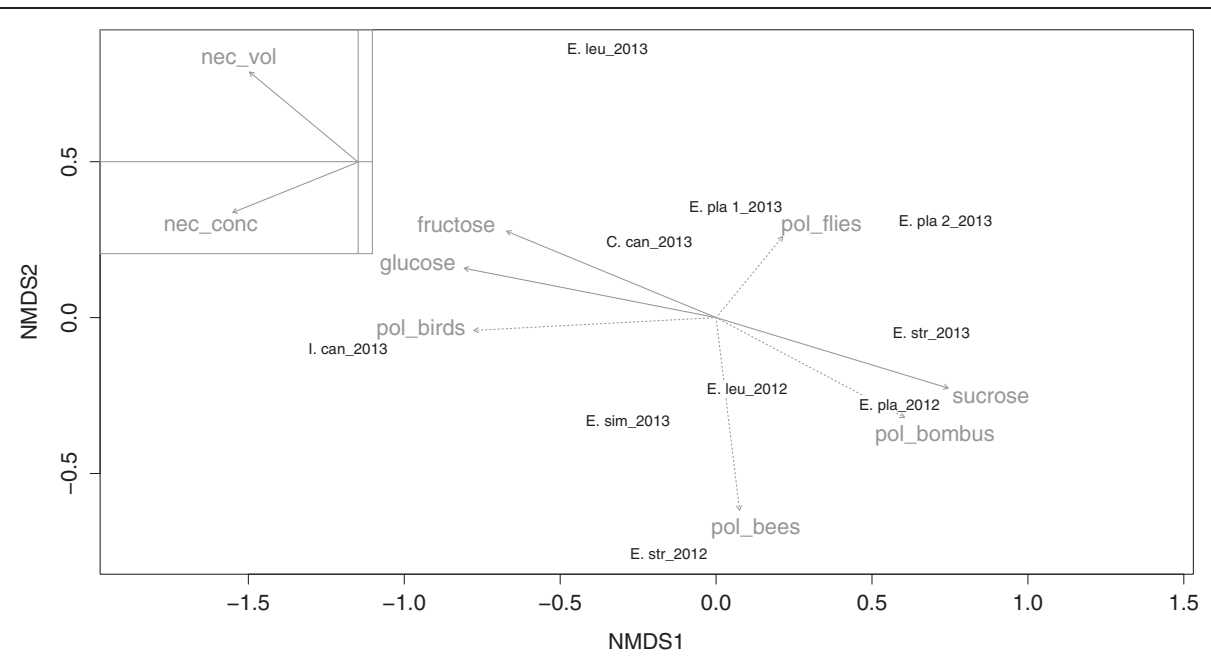

Figure 1 Ordination plot of yeast diversity. First and second axis of non-parametric multidimensional scaling of yeast diversity based on a Bray-Curtis dissimilarity matrix (stress: 0.09). The plot shows respective host-plants and sampling years. Arrows correspond to fitted nectar traits (solid lines) and flower-visitor frequencies (dotted lines): arrow directions are gradients and lengths are proportional to the correlations between variables and the ordination. Nectar volume and sugar concentration are shown separately without distortion for the sake of lucidity.

growth of only a few highly specialized ascomycetes (Figure 4). We also isolated strains of Cr. carnescens and Cys. capitatum from sugar rich nectars (Additional file 1) and showed positive growth in kinetic laboratory experiments with up to $40 \%$ sugar, which indicated their ability to grow in nectars of all studied host plants (Figure 5). Together with our results that basidiomycetous yeasts are significantly more successful in colonizing hexose dominant nectars (Figure 3), we conclude that sugar concentration alone cannot explain the composition of yeast communities in floral nectars. Additional factors, such as sugar composition (Figure 2) and a prolonged flower lifetime might be important selective filters of yeast communities in the sampled ornithophilous host plants. The latter should favor the establishment of basidiomycetes in nectar, accounting for their slower growth and extended lag phase in kinetic growth experiments in comparison to tested ascomycetous specialists (Figure 5).

(ii) Selective effects of nectar can only partially explain the reduced occurrence of ascomycetes in hexosedominant nectars. Specialized nectar yeasts are believed to grow equally well in different nectar-sugar compositions

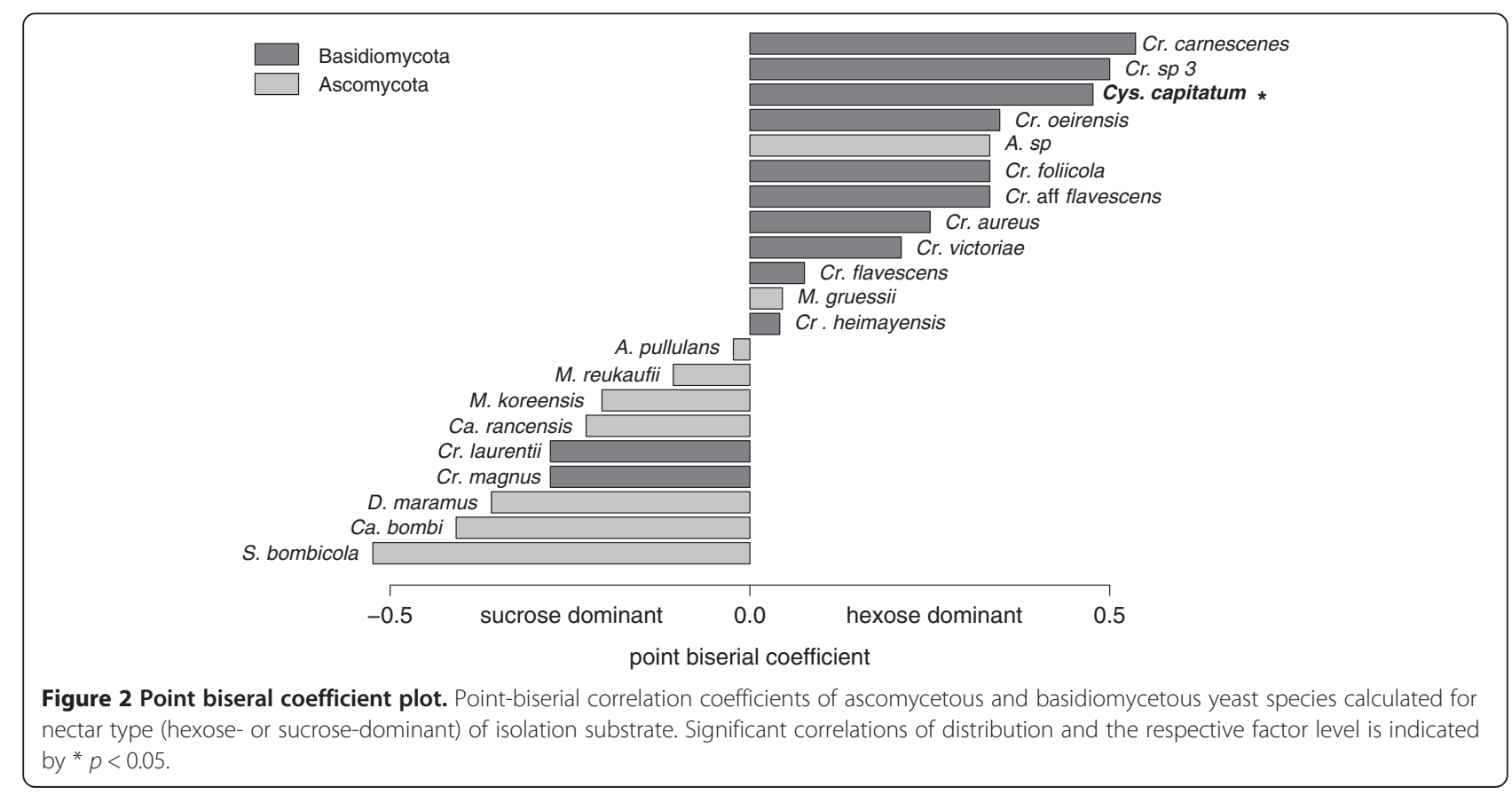




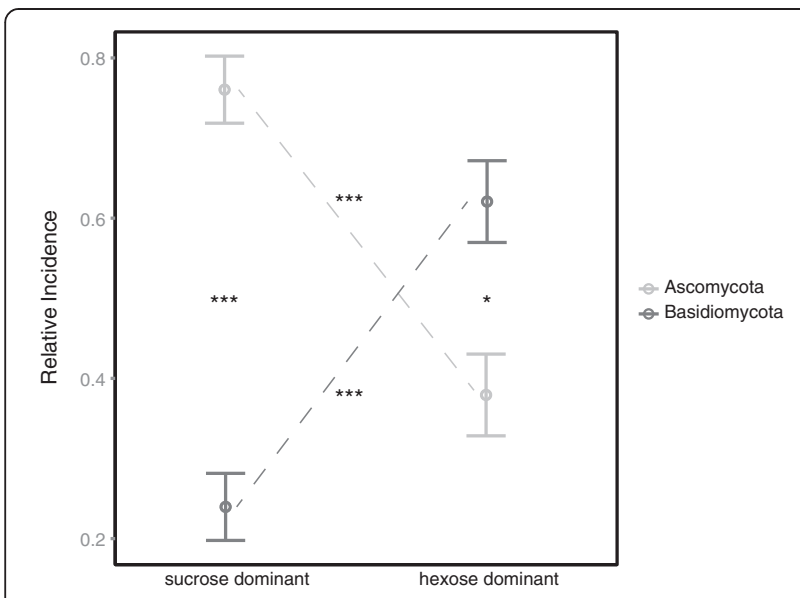

Figure 3 Relative incidences of orders. Relative incidence of ascomycetous and basidiomycetous yeast species in all sampled host plants, plotted according to respective nectar-type. Bars are standard deviations and middle points the respective mean. Significant differences (Wilcoxon pairwise tests and subsequent Holm correction) are indicated by ${ }^{*} p<0.05$, and ${ }^{* *} p<0.001$ ).
([24], Figure 2), although data on host-genotype interaction of M. reukaufii provides some support to the diversifying selection hypothesis [51] and suggests growth characteristics to be rather strain-specific.

The dominance of basidiomycetes in hexose nectars might be a result of an altered flower visitor community (Figure 4) since the visitation frequency of insects, commonly vectoring and inoculating ascomycetes $[24,28]$ is reduced. In addition, the inoculation probability of allochthonous species should be increased in ornithophilous flowers. This might be caused by the generalistic foraging behavior of nectar-feeding birds on the Canary Islands, which feed on a broad variety of resources of plant origin, such as fruits or plant tissues [52] in addition to hexose-rich nectar [45]. Since these plantrelated habitats harbor large numbers of basidiomycetous yeasts [26,27], the probability of yeast inoculation in nectar is increased by bird visitors. Indeed, South African plants, visited by passerine birds were found to harbor more yeasts (incidence and abundance) than

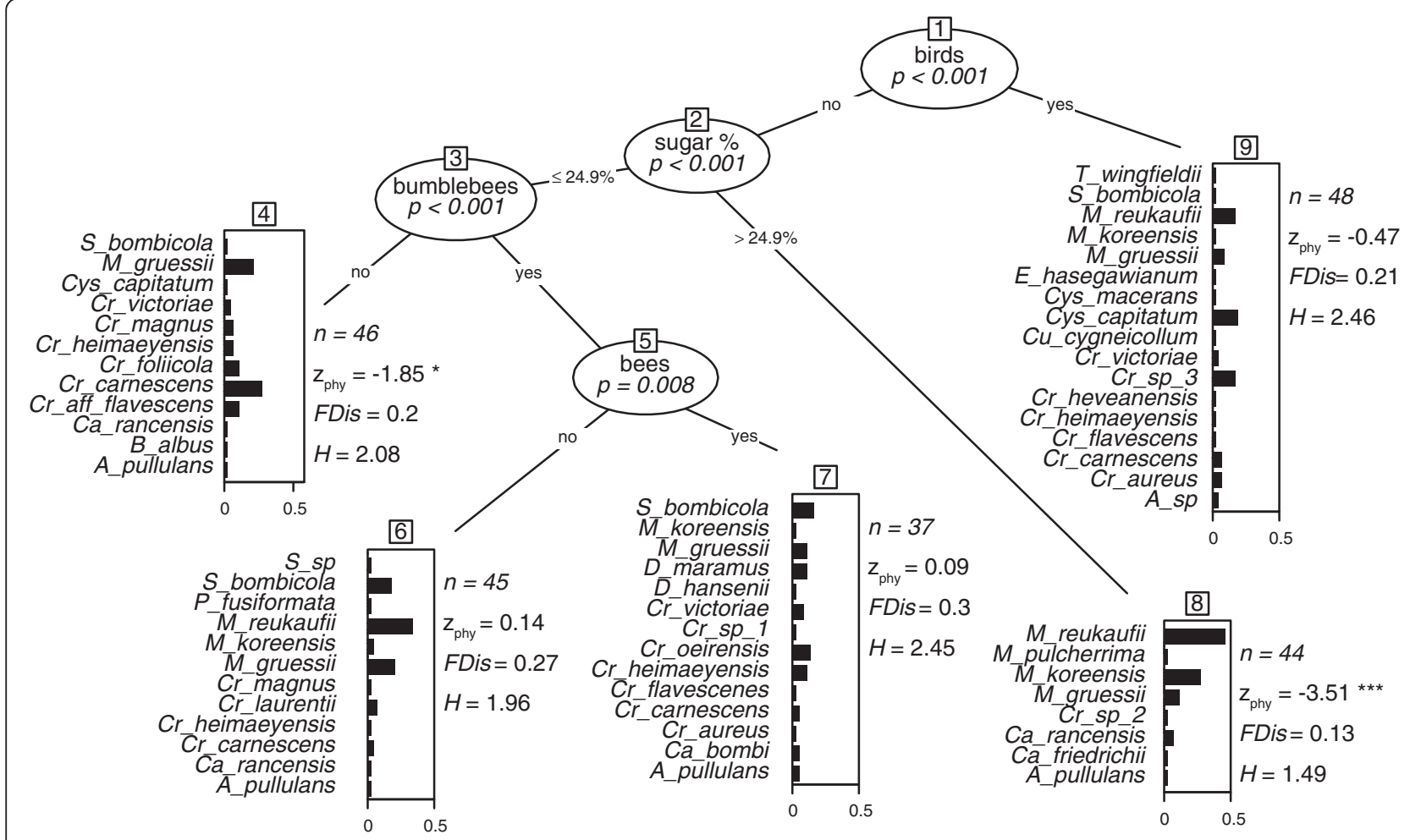

Figure 4 Partitioning tree of yeast diversity. Recursive binary partitioning tree calculated for yeast diversity predicted by nectar traits (volume + sugar concentration + nectar type), pollinator compositions (visitation frequencies of each functional group), and year. Stopping criteria were set to $a=0.01$ and minimum sizes of terminal nodes restricted to $30 \mathrm{~L}$. Nodes are numbered and respective critical splitting variable values are provided on the lines. Bar-plots show weights of species in the respective node assemblages. Light bars illustrate basidiomycetes and dark bars ascomycetes. Number of isolates (n), Functional dispersion (FDis), Shannon index (H), and mean pairwise distances compared to patterns expected under the selected null model $\left(Z_{\text {phy }}\right)$ are provided for each species assemblage. Asteriks refer to significanes $\left({ }^{*} p<0.05,{ }^{* * *} p<0.001\right)$ as calculated to the null model with default iterations and randomizations. 


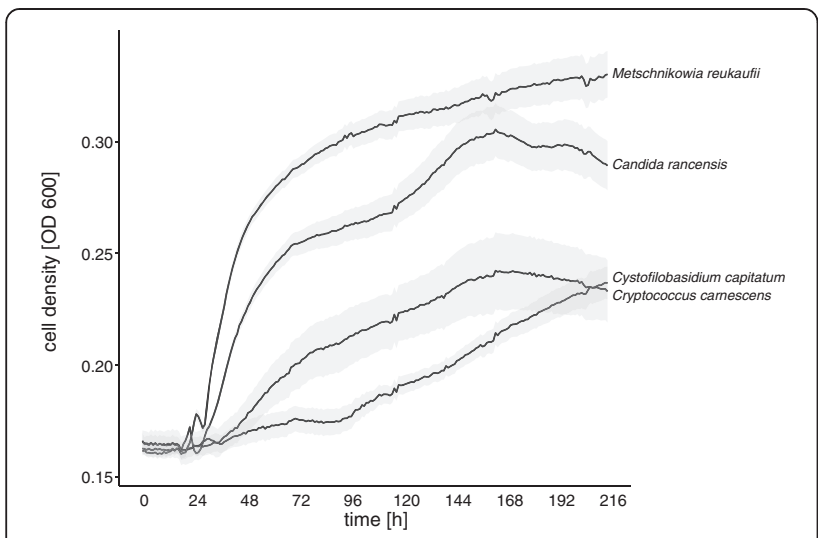

Figure $\mathbf{5}$ Growth curves of selected strains. Growth curves of selected strains in artificial nectar medium with $40 \%$ sugar concentration measured as OD 600. Shaded areas correspond to standard errors calculated of 16 replicates.

sympatric plants visited by insects, only [32]. Yeast diversity in our study is either high in flowers visited by birds or in flowers visited by bumblebees and other bees (Figure 4). Our observations mirror one common ecological law that selective pressure in the environment constrains species diversity, including microorganisms. Less strict conditions (sugar type and concentration) attract different flower visitors and allow a broader range of microbes to colonize flowers from a larger number of sources.

The shift from insect to passerine bird pollination on the Canary Islands resulted in various degrees of dependence to bird-visits, ranging from strict ornithophily in Isoplexis canariensis [42] to occasional visits by birds in Echium wildpretii [53]. As a consequence, floral adaptations to ornithophilous pollinators might be imperfect in the sense of classic pollination ecology: for example Teucrium heterophyllum is believed to be pollinated by passerine birds, despite highly concentrated sucrosedominant nectar. Taken together, these diverse and overlapping floral habitats provide a broad spectrum of available vectors and niches for microbial nectar-colonizers on a small regional scale. Our study reveals that the filter effect of nectar [28] might depend on nectar properties and on the diversity of the microbial inoculate. The pollination syndromes of the sampled host plants could in turn facilitate the inoculation and ease establishment of allochthonous microorganisms in nectars due to their species richness and overlapping diversity. These suggestions are supported by increased functional diversities of yeast communities in niches other than high-concentrated nectars (Figure 4).

Despite being combined in one ornithophilous pollinationsyndrome, passerine bird pollination in the old world and hummingbird pollination in the new world evoked different floral adaptations by plants, impeding comparisons of the diversity of nectar-dwelling microbes. Sugarconcentrations of nectars in hummingbird-flowers have been found to be elevated (25\%) in contrasts to sunbird pollinated flowers (21\%) [54]. In addition, hummingbirds commonly prefer sucrose-dominated nectars $[44,55]$ and forage on flowers with long and narrow corollas, which impede the visitation of other floral visitor-groups [56].

Indeed, nectars of hummingbird-pollinated Mimulus aurantiacus are dominated by specialized ascomycetes, such as $M$. reukaufii and Candida rancensis [18], species prevailing in sucrose-dominated flowers in our study (Figure 2). Nonetheless, Belisle et al. [57] showed that hummingbirds transport a large diversity of microfungi, including yeasts species isolated in our survey, namely C. rancensis, S. bombicola, Cr. flavescens, Cr. carnescens, and A. pullulans (Additional file 1).

\section{Basidiomycetes in nectar}

Several studies acknowledge only ascomycetes from the order Metschnikowiaceae and phylogenetically related species of the genus Candida as specialized nectar-dwelling yeasts $[24,25,28]$. The high number of isolates per species in this group underlines the expected specialization (Figure 6). The repeated isolation of a broader diversity of yeast and yeast-like species from flowers contrasts this view and suggests that additional groups of organisms might have exploited the vast number of different floral microhabitats evolved within the multitude of pollination syndromes in Angiosperms [22,58]. This hypothesis is supported by the high frequencies and withinflower abundances of 'allochthonous' species in this study (Additional file 1). Total cell densities of almost all isolated yeast species, measured as CFUs per flower show a considerable inter- and intraspecific variability, as has also been reported from other plants [18,58,59]. Interestingly, high yeast colony numbers were not restricted to fermenting ascomycetes in our study, but were also common for several basidiomycetous species in ornithophilous flowers (Additional file 1).

According to our results, Cys. capitatum and Cr. carnescens are common inhabitants of sampled flowers and may directly profit from the sampled hexose-dominant nectarenvironments (Figure 2). Brysch-Herzberg [24] also isolated Cys. capitatum from floral nectar regularly, without recognizing its potential nectar-related habit. Previously there was no evidence that Cys. capitatum might be competitive with fast-growing, fermenting ascomycetes in sugar-rich environments, due to characteristics of its former isolation habitats, such as soil $[60,61]$ and marine surface water [62]. However, recently, researchers have documented an affinity to sugar-enriched habitats by Cys. capitatum and report this species from fruits of Sorbus aucuparia L. and Rosa canina L. $[63,64]$, tree exudates ([65] and references therein), and fruiting bodies of the tree parasite Cyttaria [66]. 


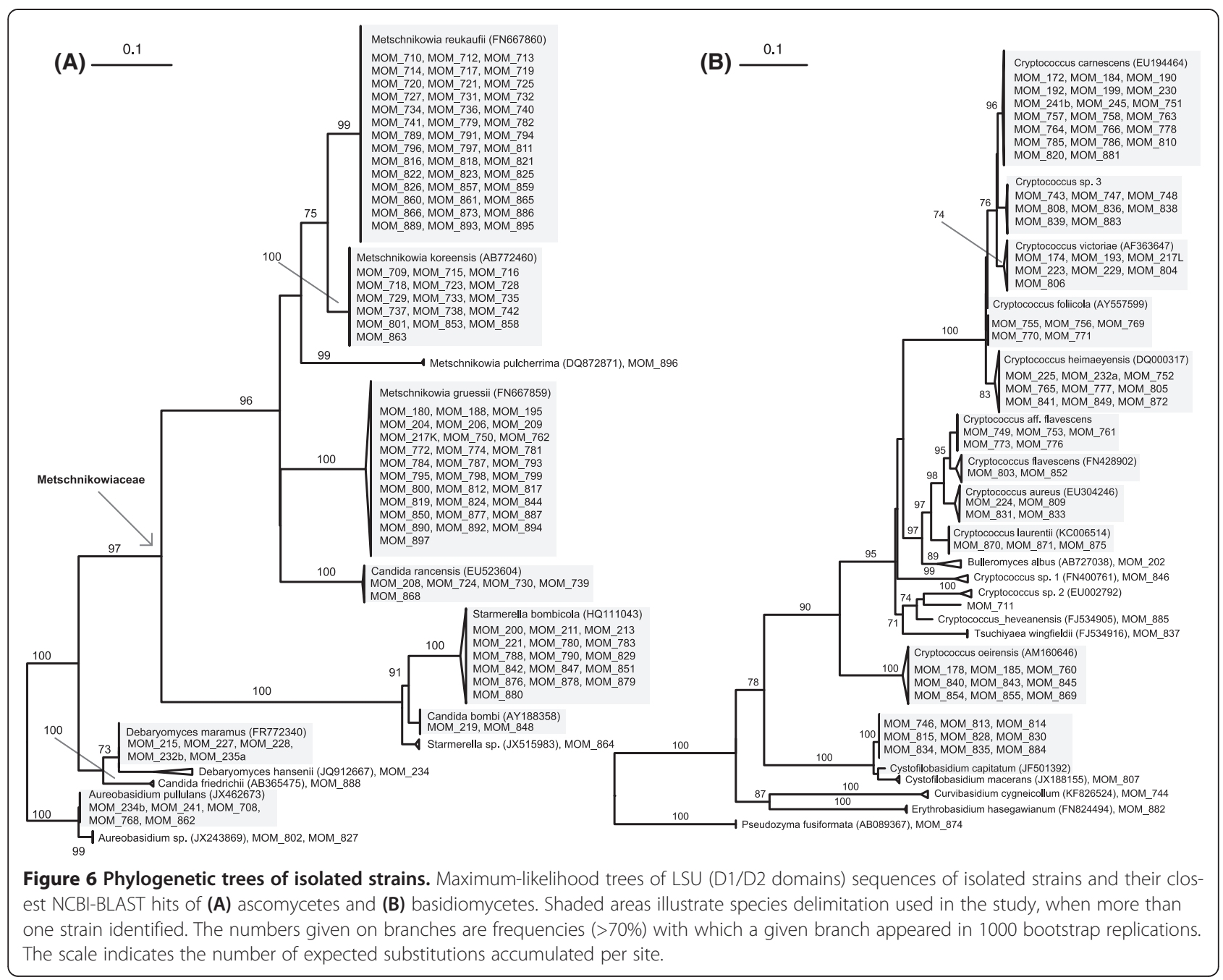

Little is known about the ecology of the second frequent basidiomycete, $\mathrm{Cr}$. carnescens, which was isolated from flowers twice $[57,67]$, and has been reported as frequent inhabitant of grapes [68] and phyllosphere in Mediterranean ecosystems [69]. Despite these plant related sources, Cr. carnescens has been understood as a pervasive species isolated from seawater, soil, and glacial ice [69]. Based on its phenotype, this yeast has been long considered a synonym of Cr. laurentii until Takashima et al. [70] demonstrated that this complex comprises several distinct and distantly related species. Although proper interpretation of both Cr. laurentii and Cr. carnescens from older studies is therefore precluded, several members of the two phylogenetic clades (Cr. laurentii and Cr. victoriae, respectively) inhabit substrates of plant origins, such as fruits and leaves [69] and were also isolated from nectar in the present study: Cr. laurentii, Cr. tephrensis, Cr. foliicola, and $\mathrm{Cr}$. heimaeyensis. The latter species was also reported from flowers before [24] but mislabeled as Cryptococcus aff. victoriae (Additional file 2).

\section{Conclusion}

In this study, we present significant trends in the community structure of nectar dwelling yeast. Nectar sugar concentration, composition, and flower visitor assemblages were identified as main selective forces. Furthermore, we reveal the previously underestimated importance of basidiomycetous yeasts as inhabitants of ornithophilous flowers with hexose dominant nectar on Tenerife. Bird-pollination on the Canary Islands clearly represents an exotic case study in the evolution of floral traits, but the provision of hexose-rich or even dominant nectar is a common phenomenon and can be found in various plants [44], e.g. related to dipteran pollination syndromes [71] or due to phylogenetic history [72]. More comprehensive data on yeast distribution across different pollination syndromes and nectar types would be clearly desirable to better comprehend the distribution, ecology, diversity and functions of basidiomycetous yeasts in floral nectar.

It is widely known that basidiomycetous and ascomycetous yeasts differ substantially in their lifestyles and 
physiological properties, suggesting different ecological strategies. While basidiomycetes have been hardly associated with nectar foraging insects, ascomycetous specialists have been almost exclusively isolated from flowers, honey pots and insects [24,73]. This leads to the conclusion that ascomycetes spend their whole life cycle inside the insect-flower system, whereas basidiomycetes might possess a broader variety of alternative substrates or even switch from saprobic to parasitic or fungicolous lifestyles [74,75]. Nonetheless, both groups highly depend on durable structures to overcome phases of transportation and rest in ephemeral nectar habitats. While the formation of ascospores in ascomycetous yeasts has been well studied, similar resistant structures of basidiomycetes in sugar-rich habitats have not been identified so far. Whether or not these ecological prerequisites together with the corresponding assimilation profiles provide basidiomycetes an advantage in colonizing nectars of ornithophilous plants requires detailed studies.

Inconsistencies in yeast incidences among years, the unbalanced experimental design, and the reliance on data from literature in this study clearly formulate the need for a more detailed and comprehensive sampling. Nonetheless, diversity patterns of nectar-borne yeasts remain stable during both years, validating our conclusions although impeding broader generalization.

\section{Experimental procedures Study sites \& plant species}

Fieldwork was conducted on the island of Tenerife in the eastern Anagar mountain region. In April 2012 a sympatric population of Echium strictum L.f., E. leucophaeum Webb ex Sprague \& Hutch., and E. plantagineum L. was studied close to Chinamada (approx. 28 $\left.33.80,-016^{\circ} 17.41^{\prime}\right)$. In May 2013, we sampled a sympatric population (approx. $\left.28^{\circ} 34.70^{\prime},-016^{\circ} 08.75^{\prime}\right)$ of E. strictum, E. leucophaeum, E. simplex DC., and E. plantagineum (1 population in scrubland and 1 population close to forest). All other studied taxa were sampled within $500 \mathrm{~m}$ of the focal population. Plant species grow in natural sclerophyllous coastal scrubland, except for Isoplexis canariensis (L.) Lindl. and Canarina canariensis (L.) Vatke, which are part of the vegetation of lower laurel forests [76]. A complete list of sampled plants can be found in Additional file 1 .

\section{Yeast isolation}

Individual flowers (flowering branches or inflorescences) in fertile female stage were carefully removed (to avoid mechanical damages) from 3 plant individuals (except for $C$. canariensis $(\mathrm{n}=6)$ and E. plantagineum $(\mathrm{n}=4))$ in the late afternoon and immediately covered in sterile plastic-bags until further processing in the lab. To account for biases due to different flower numbers of host plants, we randomly picked 40 flowers from collected plant material for nectar sampling. In adition, we covered 5 flowers of each host plant in bud stage and processed the nectar as controls. Nectar was removed from the flowers using sterile micro capillaries (Hirschmann Laborgeräte, Eberstadt, Germany) within a maximum of 4 hours after flower harvest. Total nectar volume of each flower was mixed with in $100 \mu \mathrm{l}$ of sterile tap water and streaked out on modified solid YM medium $(0.3 \% \mathrm{w} / \mathrm{v}$ Yeast extract, $0.5 \% \mathrm{w} / \mathrm{v}$ Peptone, $0.3 \% \mathrm{w} / \mathrm{v}$ Malt extract, $1 \% \mathrm{w} / \mathrm{v}$ Glucose, $2 \% \mathrm{w} / \mathrm{v}$ Agar) supplemented additionally with nectar-related sugars (1\% w/v Fructose and 1\% w/v Sucrose) and acidified with $1 \% \mathrm{v} / \mathrm{v} 80 \%$ Lactic acid (final $\mathrm{pH}=4.5$ ) to prevent bacterial growth. Plates were stored at room temperature for 4 days and then kept at lower temperature $\left(4^{\circ} \mathrm{C}\right)$ to slow down the development of molds. Plates were examined after 7 days of incubation: colonies were differentiated into macro-morphological types using dissection microscopy and the respective counts were recorded as colony forming units (CFU). One representative per plate was transferred into pure culture. All isolated strains were stored at $-80^{\circ} \mathrm{C}$ in glycerol/ glucose $(1: 1, w: w)$. Nectar samples from covered buds (controls) did not yield any fungal or yeast cultures.

\section{Yeast identification}

Pure cultures were transferred to liquid YM-medium and incubated for 48 hours at room temperature. DNA was extracted using a phenol-chloroform extraction method and the LSU ribosomal gene region (D1/D2 domains) was amplified (for detailed methods see [77]) and sequenced using the primers ITS1f or NL1 and NL4 $[78,79]$.

Sequences were edited manually and trimmed using Sequencher 5.0 following the criteria: (i) trimming no more than $25 \%$ of the sequence length until the first 25 nucleotides would contain less than 5 ambiguities, and (ii) trimming no more than $25 \%$ of the sequence length until the first 25 nucleotides would contain less than 5 nucleotides with confidences below 25. Two separate alignments for Basidiomycota and Ascomycota were created using MAFFT 7.110 [80], manually edited and curated with GBlocks allowing smaller final blocks and gap positions within it [81]. For convenience, formal classification into operational taxonomic units (OTU) was conducted using MOTHUR 1.32.1 [82] applying a 98\% cut-off value and considering also different similarity values traditionally used to delimit yeast species in ascomycetes and basidiomycetes $[18,83,84]$. Results of the OTU analysis were confirmed by morphological inspections and interpretation of phylogenetic maximum likelihood trees obtained with raxmlGUI [85] using the GTRGAMMA model and 1000 bootstrap replications (Figure 6).

Three strains with reduced sequence qualities (MOM_217, MOM_232, MOM_859) were manually inspected again with the Sequencher 5.0 software and included into an alignment 
of closely related OTUs taking into account both their morphological characterization and phylogenetic placement. Representative sequences for each OTU were identified to the species level using NCBI GenBank and MycoBank databases [86]. Sequences are stored at the EMBL nucleotide sequence database [87] and representative strains are deposited in the DSMZ, German Collection of Microorganisms and Cell Cultures (Braunschweig, Germany) (Table 1).

\section{Nectar analysis}

Standing crop nectar was removed from 25 randomly selected flowers per species and population, harvested at the same time and from the same individual plants as before. Volumes were measured with glass-capillary tubes. Sugar concentrations were estimated with a handheld refractometer (10-80\% brix, neoLab Universal, Germany). To analyze sugar compositions, 5 flowers were covered with nylon meshes in bud stage to avoid nectar contamination. Nectar samples were harvested from open flowers and stored in sterile tubes, filled with $70 \%$ Ethanol until further analysis by HPLC (see [71] for detailed methods). Nectar samples for sugar analysis from Echium leucophaeum, E. simplex, E. strictum were collected at the Botanical Garden in Berlin-Dahlem, Germany and samples of E. plantagineum at the Botanical Garden of Bonn-University, Germany, following the same procedure. Nectar sugar compositions of Canarina canariensis, Teucrium heterophyllum L'Hér., Lavatea acerifolia Cav., and Isoplexis canariensis are taken from literature [45,47].

\section{Observations of flower visitors}

Observations of floral visitors were conducted prior to nectar yeast samplings on the same individual plants. Each flower visit was counted as a new and independent event without any regard to individual visitors probing on more than one flower per plant in a row. Flower observations were undertaken in 10 min intervals with 3 researchers simultaneously, each one observing a different plant species. Focal species and individuals were changed every 30 minutes to ensure an objective threshold and to provide coverage of all plant species during all times of one day. Pollinators were pooled to functional groups as proposed by Fenster et al. [31], since we believe this classification is suitable for the objectives of this study. To increase accuracy, large plants were divided into intercepts to reduce the number of flowers observed simultaneously. Observations on Canarina canariensis and Isolplexis canariensis did not yield visitor observations, data regarding visitation rates of these species was therefore taken from Ollerton et al. [41] and Rodríguez-Rodríguez \& Valido [42], respectively. Teucrium heterophyllum is reported as generally birdpollinated $[88,89]$, and we rely on this information since no observational records were available for this plant species. Similarly, Lavetera acerifolia is considered insect-pollinated [90].

\section{Growth tests}

Growth tests were conducted in closed 96-well microplates [91] in $150 \mu \mathrm{l}$ of artificial nectar medium, consisting of yeast nitrogen base (YNB, Difco BD) and 40\% sugars mixture (Glucose, Sucrose, Fructose, 1:1:1 w/w) using Infinite 200 Pro microplate reader (Tecan Austria $\mathrm{GmbH}$, Austria). Cells from 5-day cultures were harvested from solid YM media dissolved in 1\% PBS buffer, filtered through $30 \mu \mathrm{m}$ filter (Partec $\mathrm{GmbH}$, Germany) and inoculated in the artificial nectar medium $(20$ cells per $\mu \mathrm{L}=3000$ cells per well) using BD FACSAria III cell sorter (BD Biosciences, USA) as starting cultures. Each strain was inoculated in 16 wells and a total of 32 wells were blank containing the medium only. Cultures were incubated for 10 days at $25^{\circ} \mathrm{C}$ and measured automatically every hour. Between measurements plates were incubated as static culture for 45 minutes followed by 15 minutes shaking at $1000 \mathrm{rpm}$ with $4 \mathrm{~mm}$ amplitude prior to the next absorbance measurement. Absorbance was measured at $600 \mathrm{~nm}$ every hour using the following options: multiple per well (12 reads in circle (filled) pattern) and 5 flashes in a read. Values from the reads were averaged.

\section{Data analysis}

The incidence of species was determined in all 480 flowers and organized in a presence/absence 'site"species' matrix to analyze yeast diversity. A total of 291 flowers did not yield any culturable yeast and were excluded from the analysis. To avoid biased results in the final analysis due to inflated zero counts and unequal sample sizes, yeast incidences of single flowers of each host plant and year were summarized and handled as yeast frequencies per host plant and year. Relative incidences were determined as a proportion of a particular yeast species frequency in each host plant and year. We calculated Shannon's index of diversity [92] to characterize the diversity and structure of host-specific yeast communities.

Mean nectar volume and sugar concentration for each host plant and year were standardized to account for differences in measured units. Except for nonparametric multidimensional scaling (nmds) analysis, the sugar composition was classified based on the percentages of sucrose and hexose into a factor variable (nectar type), providing 4 categories reaching from sucrose dominant to hexosedominant nectar (for details, see [55]).

Pollinator observations of individual plants were pooled to achieve 'visitation frequencies' of each functional visitor group as visits/flower/minute (v/f/min) for each host plant and year. To assess diversity of flower visitors for each 
host plant, we calculated the generalization level according to [93] as Simpson diversity index.

Mantel tests of dissimilarity matrices of yeast species frequencies (Bray-Curtis), as implemented in the R package 'vegan' [94] were used to evaluate the correlation between floral traits and flower-visitor frequencies. To create an ordination plot of yeast diversity, we applied the 'metaMDS' function for nonparametric multidimensional scaling (nmds) to the dissimilarity matrix. Floral traits and visitor frequencies were subsequently added to the nmds graphic using the implemented 'envfit' function. The function 'adonis' was used to partition the variation of yeast frequencies among factorized floral traits and visitation frequencies. To account for differences between samplings in 2012 and 2013, we constrained the subsequent permutation tests $(10,000$ replicates) to the respective sampling years. Both functions are also implemented in R package 'vegan'.

We used recursive binary partitioning to regress yeast diversity according to environmental covariates incorporating nectar traits (volume + sugar concentration + nectar type), pollinator compositions (visitation frequencies of each functional group), and sampling year. The procedure constructs unified tests for independence by means of conditional distribution of linear statistics in the permutation test framework. Stopping criteria were set to the nominal level of the conditional independence tests as $\alpha=0.01$, using a simple Bonferroni correction. The analysis was conducted with the 'ctree' function implemented in the 'party' package [95]. Subsequently, we extracted assembled yeast communities from the recursive partitioning analysis output and used the function 'ses.mpd', as implemented in the R package 'picante' [96] to test whether these assemblages represent phylogenetically clustered subsamples. The 'ses.mpd' calculates the mean pairwise distance between all species in the subsamples, based on a provided phylogenetic maximum likelihood tree, and compares phylogenetic relatedness to patterns expected under a null model, allowing for randomized community data matrix abundances within samples (maintaining species occurrence frequencies). Additionally, we calculated the functional dispersion indices for all node assemblages ('FDis' in the 'FD' package [97]) of assimilation traits for each acknowledged species.

Differences in relative incidence between ascomycetes and basidiomycetes were calculated with the Kruskal Wallis Test, using nectar type as grouping factor. Significances for single factor combinations were identified by Wilcoxon pairwise tests with subsequent Holm correction for multiple tests [98].

We calculated the point-biserial correlation coefficient to analyze the species ecological preferences [99]. The index measures the association of standardized species distributions for site groups as implemented in the $\mathrm{r}$ package 'indicspecies' [100]. All calculations were accomplished using the R framework 3.0.2. [101].

\section{Ethics statement}

All research was conducted within an appropriate ethical framework. Field work on Tenerife was conducted with permission by the Área de Medio Ambiente, Sostenibilidad Territorial y Aguas (Expte: AFF 33/13, No Sigma: 2013-00172).

\section{Availability of supporting data}

The data set supporting the results of this article is available in the Data Dryad repository, doi:10.5061/dryad.0qp3q [102].

\section{Additional files}

\section{Additional file 1: Yeast diversity. Frequencies of isolated}

ascomycetous and basidiomycetous species determined for each host plant and year are plotted in the upper line of each cell. The lower line shows mean CFUs per host plant and year and ranges in brackets. The total number of isolates per host species (column) or yeast species (line) is labeled with " $n$ ". Nucleotide sequence accession numbers, and DSMZ collection numbers are provided for each representative strain, as given by the OTU analysis.

Additional file 2: Identification of putative new yeast species. Yeast cultures were identified using nucleotide sequences of the D1/D2 domains of the large subunit (26S/28S or LSU). For species identification, the nucleotide sequences were compared with sequences deposited in the NCBI and MycolD databases, respectively. Alignments were made using the MAFFT algorithm [80]. Phylogenetic analyses were performed with MEGA 6.06 software [103]. Substitution model (Kimura two-parameter, K2 + G) was derived from model test implemented in this software. Missing data was partially (95\%) deleted. Phylogenetic placement was supported by 100 rounds of bootstrap replicates. The closest match (similarity, number of substitutions and gaps) among publically available sequences is derived from the pair-wise comparison of the two closest nucleotide sequences using blastn (NCBI) suite.

\section{Competing interests}

The authors disclose any financial or non-financial competing interests.

\section{Authors' contributions}

MM designed the experiment, conducted field and laboratory work, did the analysis, and took the lead on writing. AY and DB contributed to the study design, assisted at yeast identification, and writing of the manuscript. MW provided nectar-samples, supported the design of the fieldwork and contributed to the writing of the manuscript. DN an MN accomplished the chemical nectar analysis and contributed to the writing of the manuscript. All authors read and approved the final manuscript.

\section{Acknowledgments}

The authors like to thank O. Röhl, S. Lotze-Engelhardt, P. Testroet, and S. Mitulla for exhaustive help in the fieldwork and Dominik Schmidt for support in the lab. Sampling in Tenerife would have been impossible without Arnoldo Santos Guerra. Victoria Michael and Petra Henke (DSMZ) assisted with laboratory experiments, and Álvaro Fonseca and Marco Guerreiro (FCT/ UNL) provided their experience in identification of basidiomycetous yeasts. We thank Carlos Herrera \& Clara de Vega for critical discussions on this project and 2 anonymous reviewer for their helpful comments. The RuhrUniversity Bochum partly funded the field and lab work, and enabled the open access publication. 


\section{Author details}

'Department of Geobotany, - LS Evolution \& Biodiversity of Plants, Ruhr-University Bochum, ND 1/150 / Universitaetsstr. 150, 44780 Bochum, Germany. ${ }^{2}$ Leibniz Institute DSMZ - German Collection of Microorganisms and Cell Cultures, Braunschweig, Germany. ${ }^{3}$ Department of Life Sciences, University of Siena, Siena, Italy. ${ }^{4}$ Nees Institute for biodiversity of plants, Rheinische

Friedrich-Wilhelms-Universität, Bonn, Germany.

Received: 3 September 2014 Accepted: 15 January 2015

Published online: 01 February 2015

\section{References}

1. Garibaldi LA, Steffan-Dewenter I, Winfree R, Aizen MA, Bommarco R, Cunningham SA, et al. Wild pollinators enhance fruit set of crops regardless of honey bee abundance. Science. 2013;339:1608-11.

2. Bartomeus I, Ascher JS, Gibbs J, Danforth BN, Wagner DL, Hedtke SM, et al. Historical changes in northeastern US bee pollinators related to shared ecological traits. Proc Natl Acad Sci U S A. 2013;110:4656-60.

3. Herrera CM, Pozo MJ, Medrano M. Yeasts in nectar of an early-blooming herb: sought by bumble bees, detrimental to plant fecundity. Ecology. 2013:94:273-9.

4. Vannette RL, Gauthier M-PL, Fukami T. Nectar bacteria, but not yeast, weaken a plant-pollinator mutualism. Proc Biol Sci. 2013;280:20122601.

5. Boutroux L. Conservation des ferments alcooliques dans la nature. Ann des Sci Nat Série IV, Bot. 1884;17:145-209.

6. Jimbo T. Yeasts isolated from flower nectar. Sci Reports Tohoku Imp Univ. 1926;2:161-87.

7. Reukauf E. Die Nektarhefen. Die Kleinwelt. 1911;3:25-7.

8. Hilkenbach R. Nektarhefen - Neue Beiträge zur Kenntnis der wilden Hefen in der Natur. Kiel: Christian-Albrechts-Universitaet; 1911

9. Nadson G, Krasilnikow N. La levure du nectar des fleurs: Anthomyces reukaufii Gruess. Bull la Société Mycol Fr. 1927;43:232-44.

10. Hautmann F. Über die Nektarhefe Anthomyces Reukaufii. Arch für Protistenkd. 1921;48:213-44.

11. Martin HH. Beitrag zur Kenntnis der Morphologie und Physiologie der Nektarhefe Candida Reukaufii (Grüss) Diddens et Lodder. Arch Mikrobiol. 1954:20:141-62.

12. Babjeva IP, Gorin S. About the sporulation and the life cycle of Metschnikowia pulcherrima and M. reukaufii in the nature. Moscow Univ Her. 1973;5:82-5.

13. Herrera CM, Pozo MJ. Nectar yeasts warm the flowers of a winter-blooming plant. Proc Biol Sci. 2010;277:1827-34

14. Herrera CM, Pozo MJ, Bazaga P. Nonrandom genotype distribution among floral hosts contributes to local and regional genetic diversity in the nectar-living yeast Metschnikowia reukaufii. FEMS Microbiol Ecol. 2013;87:1-8.

15. Herrera CM, Pozo MJ, Bazaga P. Jack of all nectars, master of most: DNA methylation and the epigenetic basis of niche width in a flower- living yeast. Mol Ecol. 2012;21:2602-16.

16. Lachance M-A, Starmer WT, Rosa CA, Bowles JM, Barker JS, Janzen DH. Biogeography of the yeasts of ephemeral flowers and their insects. FEMS Yeast Res. 2001;1:1-8.

17. Herrera CM, de Vega C, Canto A, Pozo MJ. Yeasts in floral nectar: a quantitative survey. Ann Bot. 2009;103:1415-23.

18. Belisle M, Peay K, Fukami T. Flowers as islands: spatial distribution of nectar-inhabiting microfungi among plants of mimulus aurantiacus, a hummingbird-pollinated shrub. Microb Ecol. 2012;63:711-8.

19. Pozo MJ, Herrera CM, Bazaga P. Species richness of yeast communities in floral nectar of southern Spanish plants. Microb Ecol. 2011;61:82-91.

20. Zinkernagel H. Untersuchungen über Nektarhefen. Berlin: Friedrich-WilhelmsUniversitaet; 1929.

21. Wiens F, Zitzmann A, Lachance M-A, Yegles M, Pragst F, Wurst FM, et al. Chronic intake of fermented floral nectar by wild treeshrews. Proc Natl Acad Sci U S A. 2008;105:10426-31

22. Sandhu DK, Waraich MK. Yeasts associated with pollinating bees and flower nectar. Microb Ecol. 1985;11:51-8.

23. Pozo MJ, Lachance M-A, Herrera CM. Nectar yeasts of two southern Spanish plants: the roles of immigration and physiological traits in community assembly. FEMS Microbiol Ecol. 2012:80:281-93.

24. Brysch-Herzberg M. Ecology and taxonomy of yeasts associated with the plant-bumblebee mutualism in central Europe. FEMS Microbiol Ecol. 2004;50:87-100
25. Lachance M-A. Yeast biodiversity: how many and how much? In: Rosa CA, Péter G, editors. Biodivers Ecophysiol Yeasts, Ser Yeast Handb. Heidelberg: Springer Berlin; 2006. p. 1-9.

26. Last FT, Price D. Yeasts associated with living plants and their environs. In: Rose A, Harrison J, editors. Yeasts, Vol 1. London: Academic; 1969 p. 183-218.

27. Fonseca A, Inácio JJS. Phylloplane yeasts. In: Rosa CA, Peter G, editors. Biodivers Ecophysiol Yeasts, Ser Yeast Handb. Heidelberg: Springer Berlin; 2006. p. 263-301.

28. Herrera CM, Canto A, Pozo MJ, Bazaga P. Inhospitable sweetness: nectar filtering of pollinator-borne inocula leads to impoverished, phylogenetically clustered yeast communities. Proc Biol Sci. 2010;277:747-54.

29. Peay KG, Belisle M, Fukami T. Phylogenetic relatedness predicts priority effects in nectar yeast communities. Proc Biol Sci B. 2012;279:749-58.

30. Baker HG, Baker I. Chemical constituents of nectar in relation to pollination mechanisms and phylogeny. In: Nitecki HM, editor. Biochem Asp Evol Biol. Chicago, IL: Chicago University Press; 1982. p. 131-71.

31. Fenster CB, Armbruster WS, Wilson P, Dudash MR, Thomson JD. Pollination syndromes and floral specialization. Annu Rev Ecol Evol Syst. 2004;35:375-403.

32. De Vega C, Herrera CM, Johnson SD. Yeasts in floral nectar of some South African plants: quantification and associations with pollinator type and sugar concentration. South African J Bot. 2009;75:798-806.

33. Pozo MJ, de Vega C, Canto A, Herrera CM. Presence of yeasts in floral nectar is consistent with the hypothesis of microbial-mediated signaling in plant-pollinator interactions. Plant Signal Behav psb. 2009;4:1102-4.

34. Alvarez-Pérez S, Herrera CM. Composition, richness and nonrandom assembly of culturable bacterial-microfungal communities in floral nectar of Mediterranean plants. FEMS Microbiol Ecol. 2013:83:685-99.

35. Bolotin-Fukuhara M. Genomics and biodiversity in yeasts. In: Rosa C, Peter G, editors. Biodivers Ecophysiol Yeasts, Ser Yeast Handb. Heidelberg: Springer Berlin; 2006. p. 45-66 [The Yeast Handbook].

36. Sampaio JP. Utilization of low molecular weight aromatic compounds by heterobasidiomycetous yeasts: taxonomic implications. Can J Microbiol. 1999:45:491-512

37. Kurtzman CP, Fell J, Boekhout T. The Yeasts, a Taxonomic Study. 5th ed. Amsterdam: Elsevier; 2011. p. 1332-2080.

38. Phaff HJ, Miller MW, Mrak EM. The Life of Yeasts. 2nd ed. Cambridge, Massachusetts: Harvard University Press; 1978. p. 320

39. Ehlers BK, Olesen JM. The fruit-wasp route to toxic nectar in Epipactis orchids? Flora. 1997;192:223-9.

40. Vogel S. Blütenbiologische Typen als Elemente der Sippengliederung. Bot Stud. 1954;1:1-338.

41. Ollerton J, Cranmer L, Stelzer RJ, Sullivan S, Chittka L. Bird pollination of Canary Island endemic plants. Naturwissenschaften. 2009;96:221-32.

42. Rodríguez-Rodríguez MC, Valido A. Opportunistic nectar-feeding birds are effective pollinators of bird-flowers from Canary Islands: experimental evidence from Isoplexis canariensis (Scrophulariaceae). Am J Bot. 2008;95:1408-15.

43. Proctor M, Yeo P, Lack A. The Natural History of Pollination. Portland: Timber Press; 1996. p. 487

44. Baker HG, Baker I. Floral nectar sugar constituents in relation to pollinato type. In: Jones C, Little R, editors. Handb Exp Pollinat Biol. New York: Van Nostrand Reinholdt; 1983. p. 117-41.

45. Dupont YL, Hansen DM, Rasmussen JT, Olesen JM. Evolutionary changes in nectar sugar composition associated with switches between bird and insect pollination: the Canarian bird-flower element revisited. Funct Ecol. 2004;18:670-6.

46. Böhle UR, Hilger $H H$, Martin WF. Island colonization and evolution of the insular woody habit in Echium L (Boraginaceae). P Natl Acad Sci USA. 1996:93:11740-5

47. Valido A, Dupont YL, Olesen JM. Bird-flower interactions in the Macaronesian islands. J Biogeogr. 2004;31:1945-53.

48. Jacquemyn H, Lenaerts M, Brys R, Willems K, Honnay O, Lievens B. Amongpopulation variation in microbial community structure in the floral nectar of the bee-pollinated forest herb pulmonaria officinalis L. PLoS One. 2013;8:e56917.

49. Yurkov AM, Kemler M, Begerow D. Assessment of yeast diversity in soils under different management regimes. Fungal Ecol. 2012:5:24-35.

50. Heil M. Nectar: generation, regulation and ecological functions. Trends Plant Sci. 2011:16:191-200.

51. Herrera CM. Population growth of the floricolous yeast Metschnikowia reukaufii : effects of nectar host, yeast genotype and host $x$ genotype interaction. FEMS Microbiol Ecol. 2014;88:250-57. 
52. Martín A, Lorenzo JA. Aves Del Archipiélago Canario. La Laguna: Francisco Lemus; 2001.

53. Olesen JM. Floral biology of the Canarian Echium wildpretii: bird-flower or a water resource to desert bees? Acta Bot Neerl. 1988:37:509-13.

54. Nicolson SW, Fleming PA. Nectar as food for birds: the physiological consequences of drinking dilute sugar solutions. Plant Syst Evol. 2003;238:139-53.

55. Nicolson SW, Thornburg RW. Nectar chemistry. In: Nicolson SW, Nepi M, Pacini E, editors. Nectaries and Nectar. Dordrecht: Springer; 2007. p. 215-63.

56. Leins $P$, Erbar $C$. Flower and Fruit. Stuttgart: Schweizerbart'sche Verlagsbuchhandlung; 2010. p. 439.

57. Belisle M, Mendenhall CD, Oviedo Brenes F, Fukami T. Temporal variation in fungal communities associated with tropical hummingbirds and nectarivorous bats. Fungal Ecol. 2014;12:1-7.

58. Golonka AM, Vilgalys R. Nectar inhabiting yeasts in Virginian populations of silene latifolia (Caryophyllaceae) and coflowering species. Am Midl Nat. 2013;169:235-58

59. Glushakova AM, Kachalkin AV, Chernov IY. Yeasts in the Flowers of Entomophilic Plants of the Moscow Region. Microbiology. 2014;83:125-34.

60. Vishniac $\mathrm{H}$. A multivariate analysis of soil yeasts isolated from a latitudinal gradient. Microb Ecol. 2006;52:90-103.

61. Yurkov AM, Wehde T, Kahl T, Begerow D. Aboveground deadwood deposition supports development of soil yeasts. Diversity. 2012;4:453-74.

62. Fell JW, Hunter IL, Tallman AS. Marine basidiomycetous yeasts (Rhodosporidium spp. n.) with tetrapolar and multiple allelic bipolar mating systems. Can J Microbiol. 1973;19:643-57.

63. Maksimova IA, Yurkov AM, Chernov IY. Spatial structure of epiphytic yeast communities on fruits of Sorbus aucuparia L. Biol Bull. 2009;36:613-8.

64. Glushakova AM, Chernov I. Seasonal dynamics of the structure of epiphytic yeast communities. Microbiology. 2009;79:830-9.

65. Weber RWS. On the ecology of fungal consortia of spring sap-flows. Mycologist. 2006;20:140-3.

66. Libkind D, Tognetti C, Ruffini A, Sampaio JP, van Broock M. Xanthophyllomyces dendrorhous (Phaffia rhodozyma) on stromata of Cyttaria hariotii in northwestern Patagonian Nothofagus forests. Rev Argent Microbiol. 2011;43:226-32.

67. De Vega C, Herrera CM. Relationships among nectar-dwelling yeasts, flowers and ants: patterns and incidence on nectar traits. Oikos. 2012;121:1878-88.

68. Verona O, Luchetti G. Ricerche microbiologiche su di alcuni vini ed alcune uve delle marche. Boll Reg Inst Super Agrar Pisa. 1936;12:256-311.

69. Fonseca A, Boekhout T, Fell JW. Cryptococcus Vuillemin. In: Kurtzman CP, Fell JW, Boekhout T, editors. Yeasts, a Taxon study. 5th ed. Amsterdam Elsevier Amsterdam; 2011. p. 1661-737.

70. Takashima M. Three new combinations from the Cryptococcus laurentii complex: Cryptococcus aureus, Cryptococcus carnescens and Cryptococcus peneaus. Int J Syst Evol Microbiol. 2003:53:1187-94.

71. Nocentini D, Pacini E, Guarnieri M, Martelli D, Nepi M. Intrapopulation heterogeneity in floral nectar attributes and foraging insects of an ecotonal Mediterranean species. Plant Ecol. 2013;214:799-809.

72. Nicolson SW, Van Wyk B-E. Nectar sugars in proteaceae: patterns and processes. Aust J Bot. 1998:46:489-504.

73. Magyar D, Gönczöl J, Révay A, Grillenzoni F, Seijo-Coello MDC. Stauro- and scolecoconidia in floral and honeydew honeys. Fungal Divers. 2005;20:103-20.

74. Bandoni RJ. Dimorphic heterobasidiomycetes: taxonomy and parasitism. Stud Mycol. 1995:38:13-27.

75. Meirinho C, Estevinho M, Choupina A. Phylogeny and character evolution in the jelly fungi (Tremellomycetes, Basidiomycota, Fungi). Mol Phylogenet Evol. 2011;61:12-28.

76. Bramwell D, Bramwell Z. Wild Flowers of the Canary Islands. 2nd ed. Alcorcón (Madrid): Editorial Rueda SL; 2001.

77. Yurkov AM, Kemler M, Begerow D. Species accumulation curves and incidence-based species richness estimators to appraise the diversity of cultivable yeasts from beech forest soils. PLoS One. 2011;6:e23671.

78. Gardes M, Bruns T. ITS primers with enhanced specificity for basidiomycetes - application to the identification of mycorrhizae and rusts. Mol Ecol. 1993;2:113-8

79. O'Donnell K. Fusarium and its near relatives. In: Fungal Holomorph Mitotic, Meiotic Pleomorphic Speciat Fungal Syst. Wallingford: CAB International Publishing; 1993. p. 225-33.
80. Katoh K, Standley DM. MAFFT multiple sequence alignment software version 7: improvements in performance and usability. Mol Biol Evol. 2013;30:772-80.

81. Castresana J. Selection of conserved blocks from multiple alignments for their Use in phylogenetic analysis. Mol Biol Evol. 2000;17:540-52.

82. Schloss PD, Westcott SL, Ryabin T, Hall JR, Hartmann M, Hollister EB, et al. Introducing mothur: open-source, platform-independent, communitysupported software for describing and comparing microbial communities. Appl Environ Microbiol. 2009;75:7537-41.

83. Kurtzman CP, Robnett CJ. Identification and phylogeny of ascomycetous yeasts from analysis of nuclear large subunit (265) ribosomal DNA partial sequences. Antonie Van Leeuwenhoek. 1998;73:331-71.

84. Scorzetti G, Fell JW, Fonseca A, Statzell-Tallman A. Systematics of basidiomycetous yeasts: a comparison of large subunit D1/D2 and internal transcribed spacer rDNA regions. FEMS Yeast Res. 2002;2:495-517.

85. Silvestro D, Michalak I. raxmIGUI: a graphical front-end for RAxML. Org Divers Evol. 2011:12:335-7.

86. Schoch CL, Robbertse B, Robert V, Vu D, Cardinali G, Irinyi L, et al. Finding needles in haystacks: linking scientific names, reference specimens and molecular data for Fungi. Database (Oxford). 2014;2014:1-21.

87. Kanz C, Aldebert P, Althorpe N, Baker W, Baldwin A, Bates K, et al. The EMBL nucleotide sequence database. Nucleic Acids Res. 2005;33(Database issue): D29-33.

88. Mühlbauer I, Vogel S, Weber A. Blütenökologie makaronesischer Endemiten mit ornithophilen Merkmalen. Linzer biol Beitr. 2000;32:681-2.

89. Valido A, Olesen JM. Pollination on islands: examples from the Macaronesian archipelagos. In: Serrano A, Borges P, Boieiro M, Oromí P, editors. Terr arthropods Macaronesia Biodiversity, Ecol Evol. Portugal: Sociedade Portuguesa de Entomologia; 2009. p. 249-83.

90. Fuertes-Aguilar J, Ray MF, Francisco-Ortega J, Santos-Guerra A, Jansen RK Molecular evidence from chloroplast and nuclear markers for multiple colonizations of Lavatera (Malvaceae) in the Canary Islands. Syst Bot. 2002:27:74-83

91. Kurtzman CP, Fell JW, Boekhout T, Robert V. Methods for Isolation, Phenotypic Characterization and Maintenance of Yeasts. In: Kurtzman CP, Fell J, Boekhout T, editors. Yeasts, a Taxon study. 5th ed. Amsterdam Elsevier; 2011. p. 87-110.

92. Shannon CE. A Mathematical theory of communication. Bell Syst Tech J. 1948:27:379-423.

93. Sahli HF, Conner JK. Characterizing ecological generalization in plantpollination systems. Oecologia. 2006;148:365-72.

94. Oksanen J, Blanchet FG, Kindt R, Legendre P, Minchin PR, O'Hara RB, et al. vegan: Community Ecology Package. R package version 2.0-10. http://CRAN.Rproject.org/package=vegan

95. Hothorn T, Hornik K, Zeileis A. Unbiased recursive partitioning: a conditional inference framework. J Comput Graph Stat. 2006;15:651-74.

96. Kembel SW, Cowan PD, Helmus MR, Cornwell WK, Morlon H, Ackerly DD, et al. Picante: R tools for integrating phylogenies and ecology. Bioinformatics. 2010;26:1463-4.

97. Laliberté E, Legendre P. A distance-based framework for measuring functional diversity from multiple traits. Ecology. 2010;91:299-305.

98. Holm S. A simple sequentially rejective multiple test procedure. Scand J Stat. 1979;6:65-70.

99. De Cáceres M, Legendre P, Moretti M. Improving indicator species analysis by combining groups of sites. Oikos. 2010;119:1674-84.

100. De Cáceres M, Legendre P. Associations between species and groups of sites: indices and statistical inference. Ecology. 2009;90:3566-74.

101. R Core Team. R: A Language and Environment for Statistical Computing. R Foundation for Statistical Computing. 2014. [http://www.R-project.org/]

102. Mittelbach M, Yurkov AM, Nocentini D, Nepi M, Weigend M, Begerow D. Nectar sugars and bird visitation define a floral niche for basidiomycetous yeast on the Canary Islands. Gigascience 2014. http://datadryad.org/review? doi=doi:10.5061/dryad.0qp3q

103. Tamura K, Stecher G, Peterson D, Filipski A, Kumar S. MEGA6: molecular evolutionary genetics analysis version 6.0. Mol Biol Evol. 2013;30:2725-9. 